\title{
Adjuvant chemotherapy for patients with pathologic node-positive esophageal cancer after induction chemotherapy is associated with improved survival
}

\author{
Pamela Samson, MD, MPHS, ${ }^{a}$ Varun Puri, MD, MSCI, ${ }^{a}$ A. Craig Lockhart, MD, ${ }^{\mathrm{b}}$ Clifford Robinson, MD, \\ Stephen Broderick, MD, ${ }^{\mathrm{d}}$ G. Alexander Patterson, MD, ${ }^{\mathrm{a}}$ Bryan Meyers, MD, MPH, ${ }^{\mathrm{a}}$ and \\ Traves Crabtree, $\mathrm{MD}^{\mathrm{e}}$
}

\section{ABSTRACT}

Objectives: The study objectives were to identify variables associated with the use of adjuvant chemotherapy among patients with node-positive esophageal cancer who received induction therapy and to evaluate its relationship with overall survival.

Methods: Treatment data for patients with esophageal cancer receiving induction chemotherapy \pm radiotherapy and esophagectomy were abstracted from the National Cancer Data Base. Pathologic node-positive patients were dichotomized by whether they received 2 or more cycles of adjuvant chemotherapy or none. Kaplan-Meier survival curves were generated, and a Cox proportional hazards model was done to identify factors associated with overall survival.

Results: From 2006 to 2012, 3100 patients had pathologic positive nodes after induction therapy and esophagectomy. A total of 2625 patients $(84.7 \%)$ did not receive adjuvant chemotherapy, and 475 patients $(15.3 \%)$ did. N3 nodal stage was associated with an increased likelihood of receiving adjuvant chemotherapy (reference: N1, odds ratio, 1.82, 95\% confidence interval, 1.15-2.97, $P=.01$ ), whereas increasing age (by year, odds ratio, 0.97, confidence interval, 0.96$0.98, P<.001$ ), induction chemoradiation therapy (reference: induction chemotherapy, odds ratio, 0.39, confidence interval, $0.30-0.52, P<.001$ ), and increasing inpatient length of stay after esophagectomy (per day: odds ratio, 0.98 , confidence interval, 0.97-0.99, $P=.007$ ) were associated with a decreased likelihood. Patients receiving adjuvant chemotherapy had improved overall survival at each pathologic nodal stage: 31.6 months versus 22.7 months for $\mathrm{N} 1$ disease $(P<.001)$, 32.4 months versus 19.2 months for N2 disease $(P=.035)$, and 19.5 months versus 10.4 months for N3 disease $(P<.001)$. Adjuvant therapy was independently associated with decreased mortality hazard (hazard ratio, $0.69,95 \%$ confidence interval, $0.57-0.83, P<.001)$.

Conclusions: Patients receiving adjuvant chemotherapy after induction therapy and esophagectomy show a survival benefit at all positive nodal stages. Prospective studies may help further delineate this benefit. (J Thorac Cardiovasc Surg 2018;156:1725-35)

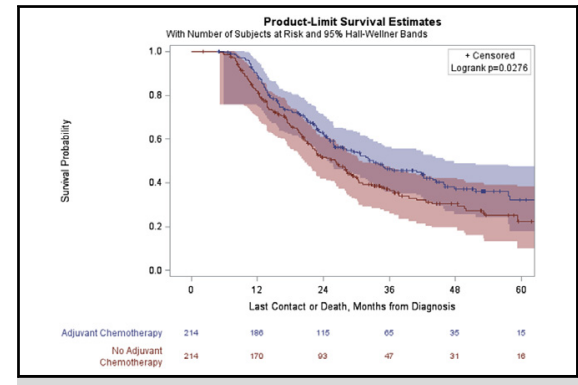

Kaplan-Meier survival analysis for patients with pathologic node-positive esophageal cancer who received induction therapy, by adjuvant chemotherapy status.

Central Message

Adjuvant chemotherapy for positive nodal disease after induction therapy and esophagectomy is independently associated with improved overall survival.

\section{Perspective}

Management of patients with esophageal cancer with positive nodal disease after induction therapy and esophagectomy is a poorly understood clinical problem. From this national database, receipt of adjuvant chemotherapy was associated with improved overall survival for patients with pathologic N1, N2, and N3.

See Editorial Commentary page 1736.
From the ${ }^{\mathrm{a}}$ Division of Cardiothoracic Surgery, Washington University in St Louis, ${ }^{c}$ Department of Radiation Oncology, Washington University in St Louis, St Louis, Mo; ${ }^{\mathrm{b}}$ Division of Medical Oncology, University of Miami, Miami, Fla; ${ }^{\mathrm{d}}$ Division of Cardiothoracic Surgery, Johns Hopkins University, Baltimore, Md; and ${ }^{\mathrm{e}}$ Division of Cardiothoracic Surgery, Southern Illinois University, Springfield, Ill.

P.S. has grant support through National Institutes of Health Cardiothoracic Surgery T32 HL07776. V.P. has grant funding through National Institutes of Health K07CA178120 and K12CA167540-02. The National Cancer Data Base is not responsible for the analytic methodology used in this study, and the conclusions drawn are solely those of the authors.
Received for publication May 24, 2016; revisions received March 13, 2018; accepted for publication May 7, 2018; available ahead of print July 24, 2018.

Address for reprints: Traves Crabtree, MD, Division of Cardiothoracic Surgery, Washington University School of Medicine, 660 S. Euclid Ave, Campus Box 8234, St Louis, MO 63110 (E-mail: crabtreet@wustl.edu).

0022-5223/\$36.00

Copyright $(\subset) 2018$ Published by Elsevier Inc. on behalf of The American Association for Thoracic Surgery

https://doi.org/10.1016/j.jtcvs.2018.05.100 


$$
\begin{aligned}
& \text { Abbreviations and Acronyms } \\
& \text { CI }=\text { confidence interval } \\
& \text { HRQL }=\text { health-related quality of life } \\
& \text { IQR }=\text { interquartile range } \\
& \text { NCDB }=\text { National Cancer Data Base } \\
& \text { OR }=\text { odds ratio } \\
& \text { pCR }=\text { pathologic complete response } \\
& \text { PEN }=\text { positive:examined node }
\end{aligned}
$$

Scanning this QR code will take you to the article title page. To view the AATS Annual Meeting Webcast, see the URL next to the webcast thumbnail.

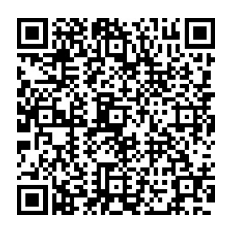

It is currently estimated that half of all patients with esophageal cancer in the United States present with local or regional disease. ${ }^{1}$ Current guidelines recommend that patients with evidence of clinically positive lymph nodes or advanced tumor depths (T2-T4a) be considered for preoperative chemoradiation therapy before esophagectomy. ${ }^{2}$ A recent review of national data demonstrated that the use of induction chemoradiation therapy has increased steadily over the past decade, with the majority of patients with locally advanced cancer (T1-3N+ and T2-3N0) receiving neoadjuvant chemoradiation therapy. ${ }^{3}$ Although induction chemoradiation therapy followed by esophagectomy has shown superior long-term survival outcomes compared with up-front esophagectomy, a clinical challenge presents itself for patients who demonstrate pathologically positive nodes after resection. ${ }^{3,4}$

A recent retrospective analysis at a single, high-volume institution has demonstrated that pathologically nodepositive patients receiving additional adjuvant chemotherapy, after induction chemoradiation and esophagectomy, experienced significant improvements in median overall survival (24 vs 18 months) and that adjuvant therapy was independently associated with a decreased mortality hazard. ${ }^{5}$ On the basis of these findings, we decided to investigate the practice patterns of adjuvant therapy after node-positive disease is discovered in patients receiving induction therapy followed by esophagectomy using the National Cancer Data Base (NCDB). Our primary aim was to identify variables independently associated with receiving adjuvant therapy after induction therapy and esophagectomy. Our secondary aim was to identify variables independently associated with overall survival in this specific population of patients.

\section{MATERIALS AND METHODS}

The NCDB Participant User File for esophageal cancer was reviewed to identify patients with esophageal cancer receiving induction chemotherapy or chemoradiation therapy, followed by esophagectomy with persistent nodal disease from 2006 to 2012. These years of analysis were chosen as the order of systemic therapy relative to surgery was routinely recorded by the NCDB starting in 2006. The NCDB is a joint effort between the American College of Surgeons Commission on Cancer and the American Cancer Society. This database currently represents approximately $70 \%$ of the malignancies treated in the United States at Commission on Cancer centers. The Participant User File contains deidentified patient and facility information, and therefore has exemption status from our Institutional Review Board.

Patient variables analyzed from the NCDB included age, race (white/ nonwhite), population type of patient's residence $(<$ or $\geq 250,000$ individuals), average income in the patient's ZIP code $(<$ or $\geq \$ 38,000$ per year), education level (percent of population in patient's ZIP code without a high school education $\geq 21 \%$ or $<21 \%$ ), insurance status (uninsured, private, Medicaid, Medicare, or other government insurance), facility type (community vs academic), and distance from the patient's ZIP code to the treatment facility in miles. As a measure of comorbidity status, the Charlson-Deyo score was abstracted and is recorded as 0,1 , or 2 or greater (not including the patient's known malignancy). Dichotomization of these categories was done per the NCDB data dictionary (http://ncdbpuf.facs. org/node/259). Patients were included for analysis if they were documented to receive induction chemotherapy or chemoradiation therapy, followed by esophagectomy, and were recorded as having 1 or more positive lymph node. A positive:examined node (PEN) ratio was calculated for each patient by dividing the number of positive lymph nodes by the number of examined lymph nodes recorded from the esophagectomy specimen. Per the NCDB variable definition, patients were noted to have received adjuvant chemotherapy if they received 2 or more cycles of systemic therapy after resection.

Descriptive statistics for continuous variables were compared using the median and interquartile range (IQR), and compared between the 2 groups using the Mann-Whitney $U$ test. Chi-square tests were used to compare categoric variables. A backward stepwise logistic regression model was created to identify variables independently associated with receiving adjuvant chemotherapy after esophagectomy in node-positive patients. Variables with a significant difference of $P$ less than .05 from univariate analysis were selected for entry into the logistic regression model. Overall survival of node-positive patients receiving adjuvant chemotherapy was compared with those who did not by using KaplanMeier analysis and the log-rank test. To account for immortal time bias (the favoring of the adjuvant therapy arm by the fact that patients in that group survived long enough to receive additional therapy after their esophagectomy), all patients with a 30 -day mortality $(\mathrm{n}=85)$ were excluded from the survival analysis. In an attempt to overcome selection and allocation bias in the receipt of adjuvant chemotherapy, a propensitymatched analysis was performed. Patients were matched on age, gender, race, insurance status, income, education level, facility type, population type, year of diagnosis, Charlson-Deyo score, induction therapy type, tumor grade, path $\mathrm{T}$ stage, path $\mathrm{N}$ stage, tumor histology, margin status, PEN ratio, readmission status, and inpatient length of stay. We calculated the propensity score using logistic regression, and patients were matched 1:1 using nearest neighbor matching with a caliper distance of 0.20 of the standard deviation of the logit of the propensity score. Postmatching diagnostics included analysis of standardized mean differences among the matching variables. Finally, a Cox proportional hazards model was created to identify variables independently associated with long-term survival. All statistical analyses were performed in SPSS, Version 22.0 (IBM Corporation, 2013, Armonk, NY). Kaplan-Meier survival curves with $95 \%$ confidence intervals (CIs) were made using SAS, Version 9.4, 2016 (SAS Institute Inc, Cary, NC). 
TABLE 1. Univariate comparison of patients with positive nodal disease after induction therapy and esophagectomy, by adjuvant chemotherapy status

\begin{tabular}{|c|c|c|c|}
\hline Variable & $\begin{array}{l}\text { Pathologic node positive, no } \\
\text { adjuvant therapy } \\
(\mathrm{n}=\mathbf{2 6 2 5 , 8 4 . 7 \% )}\end{array}$ & $\begin{array}{l}\text { Pathologic node positive, } \\
\quad \text { received adjuvant } \\
\text { therapy }(n=475,15.3 \%)\end{array}$ & $P$ value \\
\hline $\begin{array}{l}\text { Age (y) } \\
\text { Missing }=0\end{array}$ & $62(14)$ & $59(13)$ & $<.001$ \\
\hline $\begin{array}{l}\text { Gender } \\
\text { Male } \\
\text { Female } \\
\text { Missing }=0\end{array}$ & $\begin{array}{r}2266(86.3 \%) \\
359(13.7 \%)\end{array}$ & $\begin{array}{r}423(89.1 \%) \\
52(10.9 \%)\end{array}$ & .11 \\
\hline $\begin{array}{l}\text { Race } \\
\text { White } \\
\text { Nonwhite } \\
\text { Missing }=41(1.4 \%)\end{array}$ & $\begin{array}{c}2465(95.3 \%) \\
122(4.7 \%)\end{array}$ & $\begin{array}{c}456(96.8 \%) \\
15(3.2 \%)\end{array}$ & .14 \\
\hline $\begin{array}{l}\text { Greatest circle distance (miles) } \\
\text { Missing }=76(2.5 \%)\end{array}$ & $19.4(42)$ & $16.5(31.4)$ & .015 \\
\hline $\begin{array}{l}\text { Proportion in community without high school diploma } \\
\quad<21 \% \text { no high school degree } \\
\geq 21 \% \text { no high school degree } \\
\text { Missing }=77(2.6 \%)\end{array}$ & $\begin{array}{r}2235(87.5 \%) \\
318(12.5 \%)\end{array}$ & $\begin{array}{c}425(91.2 \%) \\
41(8.8 \%)\end{array}$ & .03 \\
\hline $\begin{array}{l}\text { Income } \\
\qquad \$ 38,000 \\
\quad<\$ 38,000 \\
\text { Missing }=77(2.6 \%)\end{array}$ & $\begin{array}{r}2199(86.1 \%) \\
354(13.9 \%)\end{array}$ & $\begin{array}{c}420(90.1 \%) \\
46(9.9 \%)\end{array}$ & .02 \\
\hline $\begin{array}{l}\text { Population type } \\
\text { Metropolitan county } \\
\text { Urban county } \\
\text { Rural county } \\
\text { Missing }=142(4.7 \%)\end{array}$ & $\begin{array}{c}2013(80.7 \%) \\
428(17.2 \%) \\
53(2.1 \%)\end{array}$ & $\begin{aligned} 380 & (82.8 \%) \\
72 & (15.7 \%) \\
7 & (1.5 \%)\end{aligned}$ & .50 \\
\hline $\begin{array}{l}\text { Insurance status } \\
\text { Uninsured } \\
\text { Private insurance } \\
\text { Medicaid } \\
\text { Medicare } \\
\text { Other government insurance } \\
\text { Missing }=29(1.0 \%)\end{array}$ & $\begin{array}{c}62(2.4 \%) \\
1356(52.2 \%) \\
137(5.3 \%) \\
1010(38.8 \%) \\
35(1.3 \%)\end{array}$ & $\begin{array}{c}16(3.4 \%) \\
300(63.7 \%) \\
24(5.1 \%) \\
122(25.9 \%) \\
9(1.9 \%)\end{array}$ & $<.001$ \\
\hline $\begin{array}{l}\text { Charlson-Deyo score } \\
0 \\
1 \\
\geq 2 \\
\text { Missing }=0\end{array}$ & $\begin{array}{c}1966(74.9 \%) \\
530(20.2 \%) \\
129(4.9 \%)\end{array}$ & $\begin{array}{c}375(78.9 \%) \\
82(17.3 \%) \\
18(3.8 \%)\end{array}$ & .16 \\
\hline $\begin{array}{l}\text { Facility type } \\
\text { Academic } \\
\text { Community } \\
\text { Missing }=0\end{array}$ & $\begin{array}{l}1458(55.6 \%) \\
1162(44.4 \%)\end{array}$ & $\begin{array}{l}246(51.9 \%) \\
228(48.1 \%)\end{array}$ & .13 \\
\hline $\begin{array}{l}\text { Histology } \\
\text { Adenocarcinoma } \\
\text { Squamous cell } \\
\text { Missing }=344(11.4 \%)\end{array}$ & $\begin{array}{r}1961(83.9 \%) \\
377(16.1 \%)\end{array}$ & $\begin{array}{c}372(91.6 \%) \\
34(8.4 \%)\end{array}$ & $<.001$ \\
\hline $\begin{array}{l}\text { Neoadjuvant type } \\
\text { Chemotherapy } \\
\text { Chemoradiation therapy } \\
\text { Missing }=0\end{array}$ & $\begin{array}{r}346(13.2 \%) \\
2279(86.8 \%)\end{array}$ & $\begin{array}{l}131(27.6 \%) \\
344(72.4 \%)\end{array}$ & $<.001$ \\
\hline
\end{tabular}


TABLE 1. Continued

\begin{tabular}{|c|c|c|c|}
\hline Variable & $\begin{array}{c}\text { Pathologic node positive, no } \\
\text { adjuvant therapy } \\
(\mathbf{n}=\mathbf{2 6 2 5}, \mathbf{8 4 . 7} \%)\end{array}$ & $\begin{array}{c}\text { Pathologic node positive, } \\
\text { received adjuvant } \\
\text { therapy }(n=475,15.3 \%)\end{array}$ & $P$ value \\
\hline \multicolumn{4}{|l|}{ Path T } \\
\hline T0 & $213(8.4 \%)$ & $29(6.4 \%)$ & .15 \\
\hline $\mathrm{T} 1$ & $299(11.8 \%)$ & $53(11.6 \%)$ & \\
\hline $\mathrm{T} 2$ & $441(17.4 \%)$ & $83(18.2 \%)$ & \\
\hline $\mathrm{T} 3$ & $1508(59.6 \%)$ & $285(62.6 \%)$ & \\
\hline $\mathrm{T} 4$ & $68(2.7 \%)$ & $5(1.1 \%)$ & \\
\hline \multicolumn{4}{|l|}{ Missing $=113(3.7 \%)$} \\
\hline \multicolumn{4}{|l|}{ Pathologic N } \\
\hline N1 & $2160(82.3 \%)$ & $355(74.7 \%)$ & $<.001$ \\
\hline $\mathrm{N} 2$ & $352(13.4 \%)$ & $74(15.6 \%)$ & \\
\hline N3 & $113(4.3 \%)$ & $46(9.7 \%)$ & \\
\hline \multicolumn{4}{|l|}{ Missing $=0$} \\
\hline Positive margins $(\geq \mathrm{R} 1)$ & $297(11.5 \%)$ & $59(12.7 \%)$ & .45 \\
\hline \multicolumn{4}{|l|}{ Missing $=49(1.6 \%)$} \\
\hline Tumor size (mm) & $40(30)$ & $40(27)$ & .09 \\
\hline \multicolumn{4}{|l|}{ Missing $=832(26.8 \%)$} \\
\hline No. of nodes examined & $14(11)$ & $15(13)$ & .008 \\
\hline \multicolumn{4}{|l|}{ Missing $=46(1.5 \%)$} \\
\hline No. of positive nodes & $2(3)$ & $3(5)$ & $<.001$ \\
\hline \multicolumn{4}{|l|}{ Missing $=0$} \\
\hline PEN ratio & $0.17(0.24)$ & $0.20(0.29)$ & .016 \\
\hline \multicolumn{4}{|l|}{ Missing $=46(1.5 \%)$} \\
\hline Inpatient length of stay (d) & $10.0(6)$ & $9(6)$ & .008 \\
\hline \multicolumn{4}{|l|}{ Missing $=356(11.5 \%)$} \\
\hline 30-d readmission & $200(7.9 \%)$ & $30(6.6 \%)$ & .32 \\
\hline Missing $=102(3.4 \%)$ & & & \\
\hline
\end{tabular}

$P E N$, Positive:examined node

\section{RESULTS}

From 2006 to 2012, 9307 patients received induction therapy followed by esophagectomy and were recorded as having at least 1 lymph node sampled. A total of 3100 $(33.3 \%)$ of these patients were documented as having at least 1 positive lymph node. Specifically, 2515 patients $(81.1 \%)$ had pathologic N1 disease, 426 patients (13.7\%) had N2 disease, and 159 patients $(5.1 \%)$ had N3 disease. Of these 3100 node-positive patients, $2625(84.7 \%)$ did not go on to receive additional adjuvant therapy, and 475 $(15.3 \%)$ did. Clinical characteristics of patients receiving adjuvant chemotherapy are demonstrated in Table 1. Volume of cases per facility for this cohort was divided into quartiles, with the highest quartile recording more than 14 cases per year. There was no difference in receipt of adjuvant chemotherapy between the highest quartile of esophagectomy volume and the lower 3 quartiles $(16.8 \%$ vs $15.4 \%$, respectively, $P=.36$ ).
On logistic regression modeling, variables that were independently associated with an increased likelihood of receiving adjuvant chemotherapy included adenocarcinoma histology (odds ratio [OR], 2.03, 95\% CI, 1.33-3.09, $P=.001$, reference: squamous cell) and increasing pathologic $\mathrm{N}$ status (N3: OR, 1.82, 95\% CI, 1.15-2.97, $P=.01$, reference N1). Pathologic N2 status approached significance (OR, 1.35, 95\% CI, 0.98-1.87, $P=.06$, reference N1). Variables independently associated with a decreased likelihood of receiving adjuvant chemotherapy included increasing age (per year: OR, 0.97, 95\% CI, $0.96-0.98, P<.001$ ), use of induction chemoradiation therapy (OR, $0.39,95 \% \mathrm{CI}, 0.30-0.52, P<.001$, reference: induction chemotherapy), and increasing length of inpatient stay (per day: OR, 0.98, 95\% CI, 0.97-0.99, $P=.007$ ) (Table 2).

To further investigate the strong association between induction therapy type (chemotherapy alone vs 
TABLE 2. Backward stepwise logistic regression model for variables independently associated with receiving adjuvant chemotherapy status for patients with positive nodal disease after induction therapy and esophagectomy

\begin{tabular}{lcc}
\hline \multicolumn{1}{c}{ Variable } & Odds ratio $(\mathbf{9 5} \% \mathbf{C I})$ & $\boldsymbol{P}$ value \\
\hline Age (per year increase) & $0.97(0.96-0.98)$ & $<.001$ \\
Adenocarcinoma histology & $2.03(1.33-3.09)$ & .001 \\
Neoadjuvant chemoradiation & $0.39(0.30-0.52)$ & $<.001$ \\
Pathologic N status (ref: N1) & & \\
N2 & $1.35(0.98-1.87)$ & .06 \\
N3 & $1.85(1.15-2.97)$ & .01 \\
Length of stay (per day & $0.98(0.97-0.99)$ & .007 \\
$\quad$ increase) & & \\
\hline
\end{tabular}

$\overline{\text { Variables were selected for model entry if } P<.05 \text { on univariate analysis. These are the }}$ final variables that were independently associated with receipt of adjuvant chemotherapy. $\mathrm{CI}$, Confidence interval.

chemoradiation) and adjuvant therapy status in this nodepositive population, we compared short-term outcomes and pathologic disease burden of patients by induction therapy type. Of note, there was no significant difference in length of stay (12.5 days \pm 10.8 for patients receiving induction chemotherapy vs 13.2 days \pm 11.4 for patients receiving induction chemoradiation, $P=.23$ ) or 30-day readmission status $(6.6 \%$ vs $7.9 \%$, respectively, $P=.33)$. In terms of residual disease burden, patients receiving induction chemoradiation were significantly less likely to have positive margins ( $\geq \mathrm{R} 1$ resection) than patients receiving induction chemotherapy $(10.9 \%$ vs $16.0 \%, P=.002)$ and had a lower mean number of positive lymph nodes $(3.0 \pm 3.0 \mathrm{vs}$ $4.1 \pm 4.7, P<.001)$. Decreases in pathologic stage grouping and PEN ratio approached but did not reach significance.

Median follow-up time was 21 months (IQR, 22 months). Of note, $84.8 \%$ of patients died before the median followup time was reached, and $31.9 \%$ of patients were lost to follow-up. On survival analysis, node-positive patients who received adjuvant chemotherapy demonstrated a significant improvement in median overall survival compared with those who did not: 30.8 months (IQR, 16-63.6) versus 23.0 months (IQR 12.9-45.8) $(P<.001)$. Kaplan-Meier curve and log-rank test for these 2 groups are shown in Figure 1. This increase in median overall survival remained true for patients who were pathologically node positive but had R0 resections: 33.3 months (IQR, 17.0-74.0) for those who did receive adjuvant chemotherapy versus 24.0 months (IQR, 13.5-49.8) for patients who did not receive adjuvant chemotherapy $(P<.001)$ (Kaplan-Meier and log rank, Figure 2). When R0, node-positive patients were compared by histologic subtype, patients with adenocarcinoma (33.3 months [IQR, 17.2, not reached] versus 25.3 months [IQR, 13.7-50.0], $P<.001)$ and patients with squamous

Product-Limit Survival Estimates

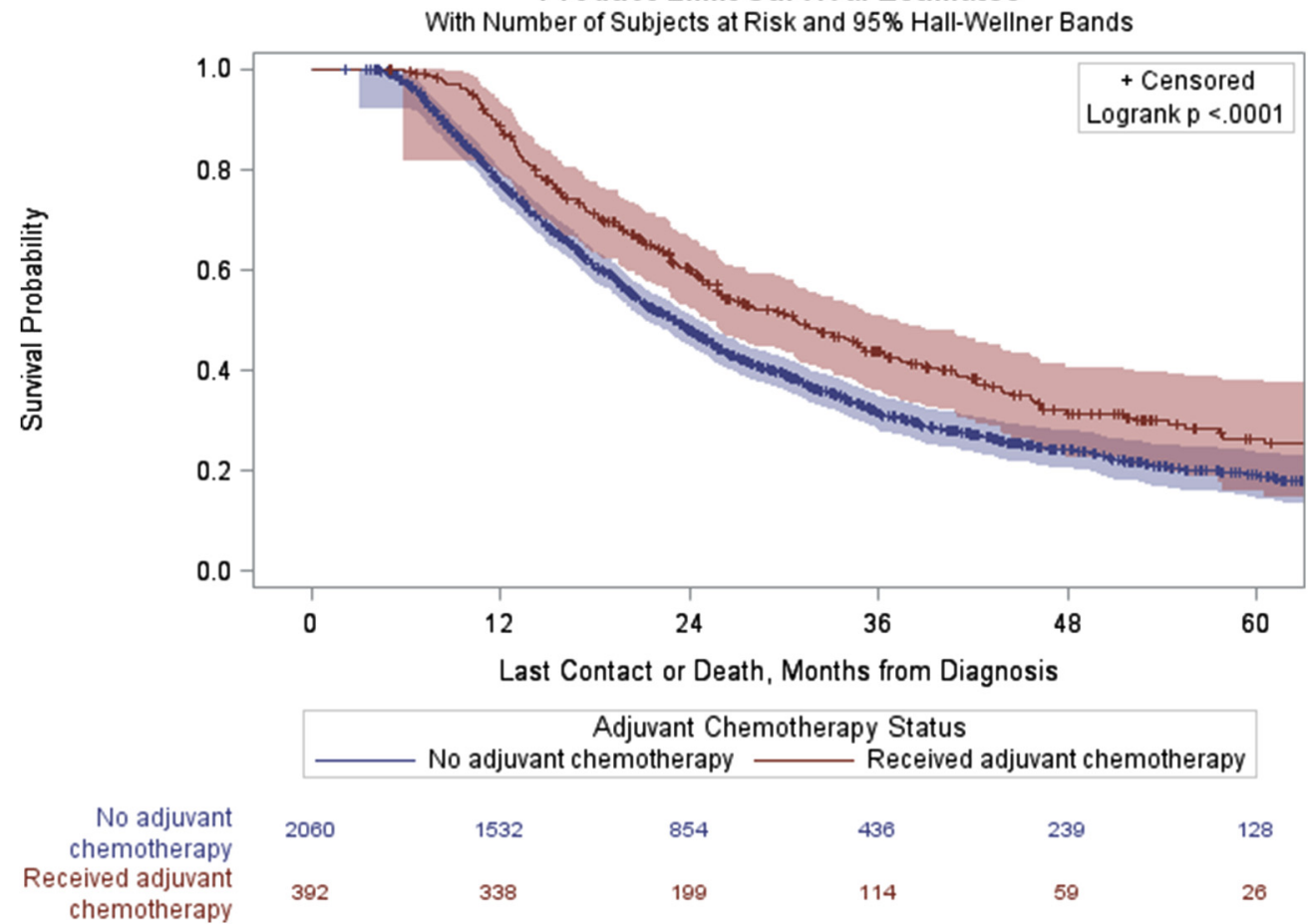

FIGURE 1. Kaplan-Meier analysis for all pathologically node-positive patients who received induction chemotherapy/chemoradiation therapy and esophagectomy, by adjuvant status. 


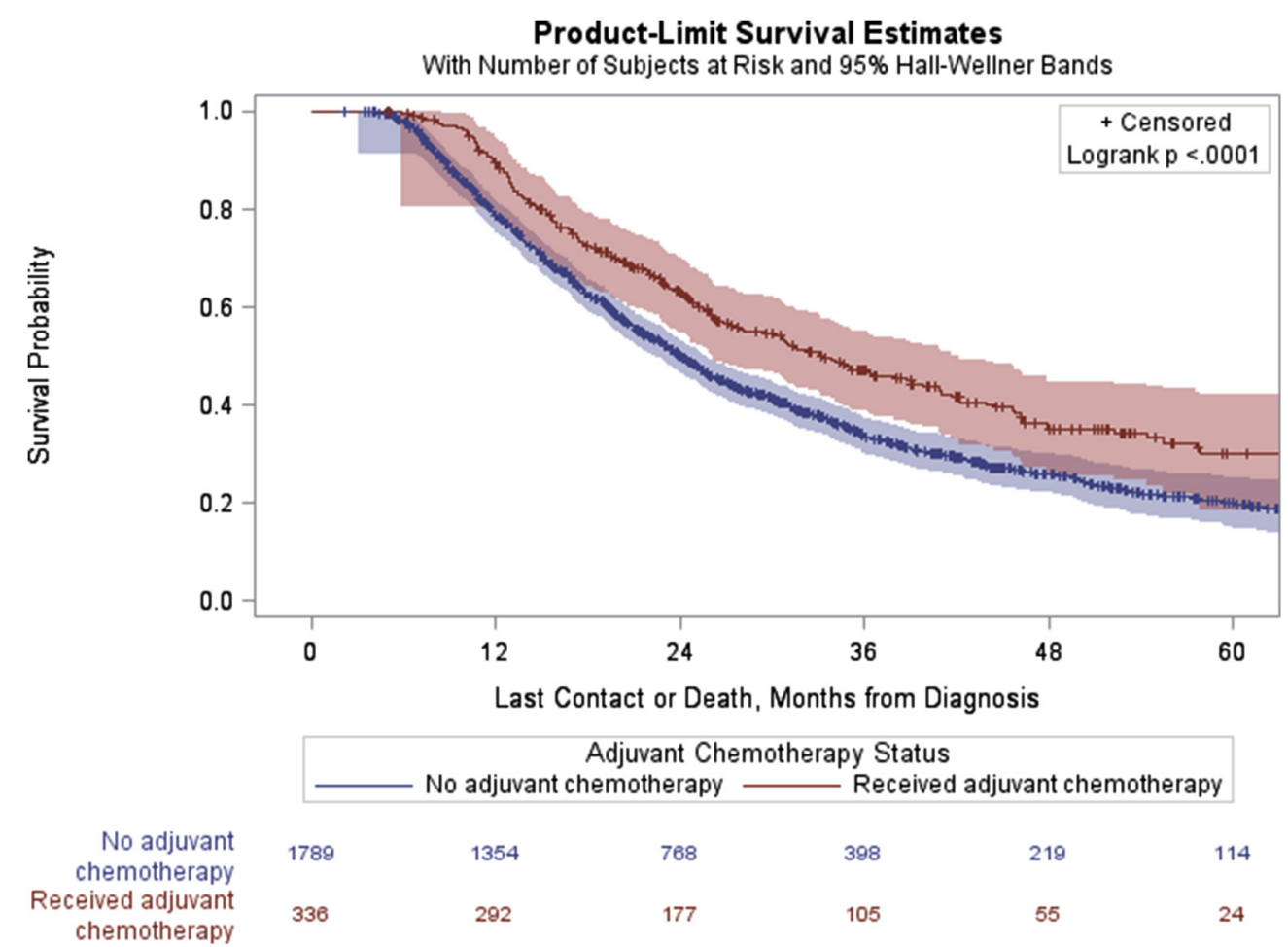

FIGURE 2. Kaplan-Meier analysis for pathologically node-positive patients with negative surgical margins (R0 resection) who received induction chemotherapy/chemoradiation therapy and esophagectomy, by adjuvant status.

cell carcinoma (45.8 months [IQR, 15.6, not reached] versus 22.1 months [IQR, 13.1-47.1], $P=.03$ ) receiving adjuvant therapy both experienced improvements in overall survival compared with those who did not, respectively. Adjuvant chemotherapy resulted in improved overall survival for each pathologic nodal stage: pathologic N1 disease, 31.6 months (IQR, 17.0-71.7) versus 23.6 months (IQR, 13.5-48.4) $(P<.001)$ (Figure 3); pathologic N2 disease, 32.4 months (IQR, 15.8, not reached) versus 20.4 months (IQR, 11.1-46.8) $(P=.035)$; and pathologic $\mathrm{N} 3$ disease, 19.5 months (IQR, 12.0-30.7) versus 11.9 months (IQR 7.8-26.9) $(P=.04)$. This survival benefit was also seen for patients with a low burden of nodal disease, including patients with 1 positive lymph node (39.8 months [IQR, 18.3-74.0] vs 27.4 months [IQR, 15.2-59.5], $P=.01$ ) and patients with a PEN ratio less than 0.10 (45.8 months [IQR, 25.2, not reached] vs 31.0 months [IQR, 17.1-63.7], $P=.004$ ).

Of 3015 patients, 1445 had complete data available for matching (detailed in the "Materials and Methods" section). Of these 1445 patients, $1230(85.1 \%)$ did not receive adjuvant chemotherapy, and $215(14.8 \%)$ did receive adjuvant chemotherapy. A total of 214 of 215 patients $(99.5 \%)$ who received adjuvant therapy were matched to 214 of 1445 patients $(14.8 \%)$ who did not receive adjuvant therapy. No variables displayed a standardized mean difference greater than 0.15 , indicating a good match. Upon propensity score matching, median overall survival remained significantly higher for patients receiving adjuvant therapy compared with those matched patients who did not: 33.1 months (IQR, 16.1-74.0) versus 26.2 months (IQR, 13.8-59.3) $(P=.03)$. Kaplan-Meier curves for these 2 populations with log-rank test are shown in Figure 4.

A Cox proportional hazards model was fitted to identify variables independently associated with overall mortality hazard (Table 3). Of the variables evaluated, receipt of adjuvant chemotherapy was associated with the largest reduction in mortality (HR, $0.71,95 \%$ CI, 0.59-0.87, $P=.001$ ), while controlling for other patient and tumor factors. The other variables independently associated with a decrease in mortality included receiving treatment at an academic facility (HR, 0.84, 95\% CI, 0.74-0.95, $P=.007$ ) and adenocarcinoma histology type (HR, 0.83, 95\% CI, 0.70-0.99, $P=.04$ ).

\section{DISCUSSION}

Current trends in esophageal cancer care demonstrate that the majority of surgical candidates with locally advanced disease are receiving induction chemotherapy or chemoradiation therapy, but one third continue to have pathologically positive nodal disease. Despite the frequency 


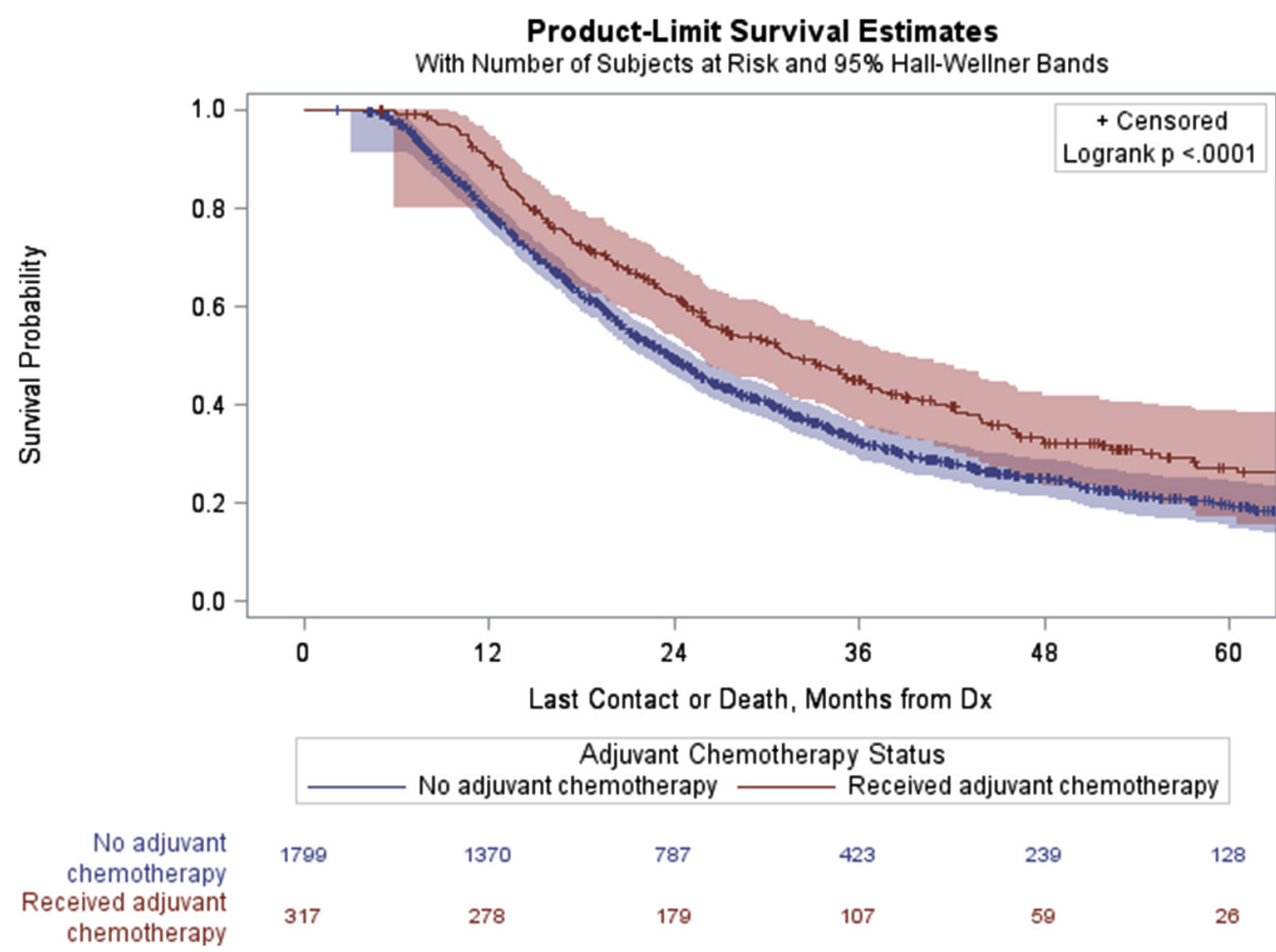

FIGURE 3. Kaplan-Meier analysis for pathologic N1 patients who received induction chemotherapy/chemoradiation therapy and esophagectomy, by adjuvant status.

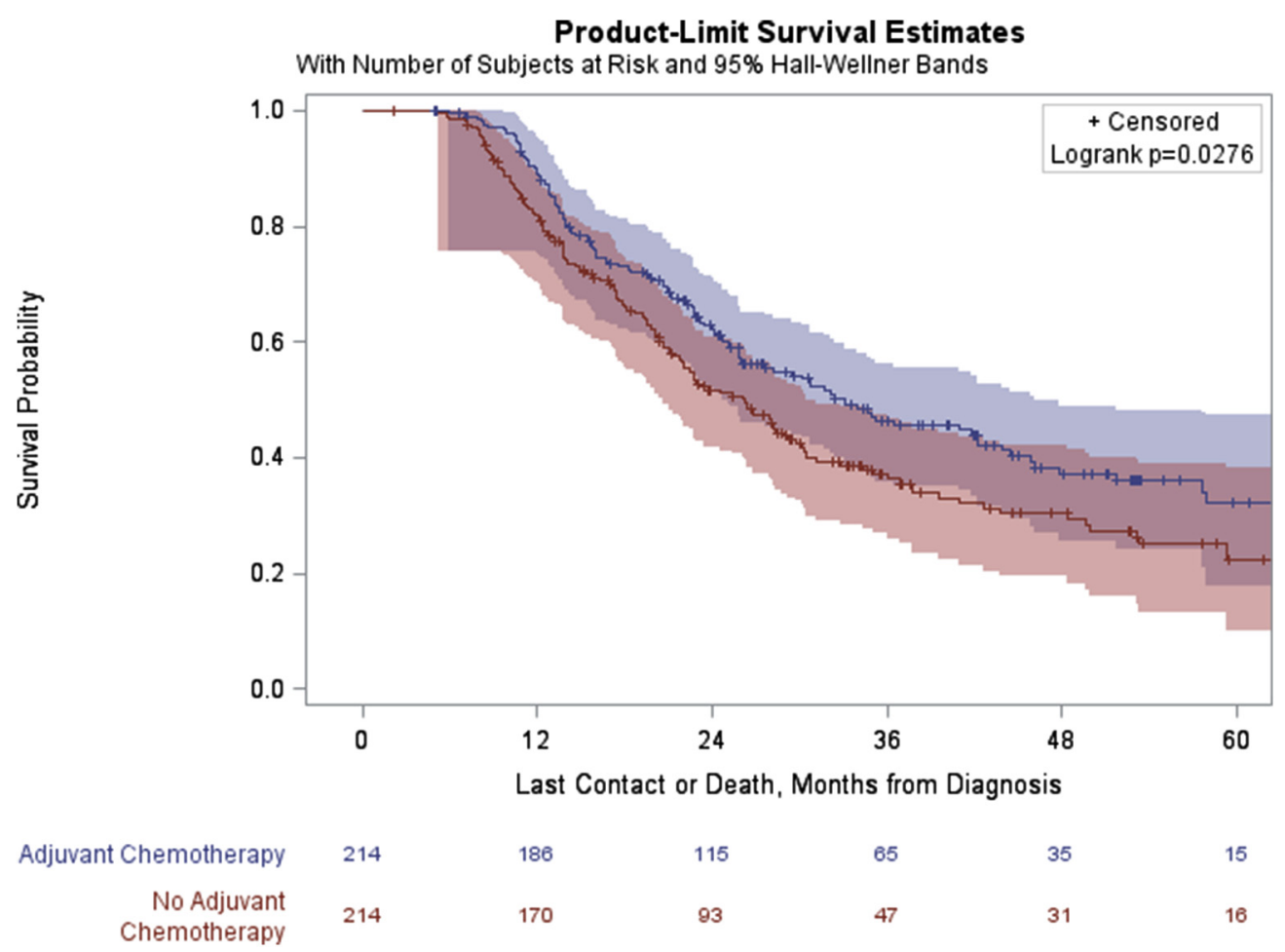

FIGURE 4. Kaplan-Meier analysis for propensity matched node-positive esophageal cancer patients who received induction chemotherapy/chemoradiation therapy and esophagectomy, by adjuvant status. 
TABLE 3. Cox-proportional hazards model for variables independently associated with an overall survival in patients with pathologically node-positive esophageal cancer who completed induction therapy and esophagectomy

\begin{tabular}{lcc}
\hline \multicolumn{1}{c}{ Variable } & Hazard ratio $(\mathbf{9 5} \% \mathbf{C I})$ & $\boldsymbol{P}$ value \\
\hline Age (by year) & $1.02(1.01-1.02)$ & $<.001$ \\
Income $<\$ 38,000 / \mathrm{y}$ & $1.52(1.28-1.81)$ & $<.001$ \\
\hline Academic cancer center & $0.84(0.74-0.95)$ & .007 \\
Induction chemoradiation & $1.25(1.06-1.49)$ & .01 \\
$\quad$ therapy (ref: induction & & \\
$\quad$ chemotherapy) & & .04 \\
Adenocarcinoma histology & $0.83(0.70-0.99)$ & \\
Tumor grade (ref: well & & .78 \\
$\quad$ differentiated) & $1.08(0.74-1.56)$ & .03 \\
$\quad$ Moderately differentiated & $1.52(1.05-2.20)$ & .05 \\
$\quad$ Poorly differentiated & $1.67(0.99-2.80)$ & .53 \\
$\quad$ Undifferentiated & & $<.001$ \\
\hline Pathologic N stage (ref: N1) & $1.08(0.86-1.35)$ & $<.001$ \\
$\quad$ N2 & $2.10(1.49-2.95)$ & $<.001$ \\
$\quad$ N3 & $1.61(1.32-1.96)$ & .003 \\
\hline Positive margin status $(\geq \mathrm{R} 1)$ & $1.01(1.01-1.02)$ & .001 \\
\hline Inpatient length of stay (per & & \\
increasing day) & $1.40(1.12-1.75)$ & \\
\hline 30-d readmission & $0.71(0.59-0.87)$ & \\
\hline Adjuvant chemotherapy & & \\
\hline
\end{tabular}

Variables entered into the model included age, gender, race, income and education level in patient's ZIP code, distance from the treatment center, treatment center type, population type, histology, tumor grade, induction therapy type, margin status, pathologic $\mathrm{T}$ and $\mathrm{N}$ stage, length of inpatient stay, 30-day readmission status, and adjuvant therapy status. $\mathrm{CI}$, Confidence interval.

of this clinical scenario, little evidence exists regarding the role of additional adjuvant chemotherapy. In this NCDB analysis of patients with pathologically node-positive esophageal cancer who received induction therapy and esophagectomy, we found that a minority of patients go on to receive additional adjuvant chemotherapy $(15 \%)$. However, pathologically node-positive patients receiving additional chemotherapy experienced a significant and substantial improvement in median overall survival, and this improvement in survival was independent of other patient and tumor characteristics.

This NCDB analysis has several novel findings that differ from a recent retrospective single-institution analysis that examined outcomes among patients receiving adjuvant therapy for positive nodal disease after induction therapy and esophagectomy. ${ }^{5}$ In the Washington University experience of 101 node-positive patients who had undergone induction therapy (with $>90 \%$ receiving induction chemoradiation therapy) followed by esophagectomy, a substantially higher proportion received adjuvant chemotherapy: approximately $44 \%$ versus $15 \%$ in our national series. ${ }^{5}$ Although our NCDB analysis did identify age as independently associated with adjuvant chemotherapy status $(3 \%$ decrease in the likelihood of receiving adjuvant chemotherapy for every year increase), both analyses found no difference in adjuvant uptake based on the patient's comorbidity status. ${ }^{5}$ Furthermore, both our analysis and the analysis by Brescia and coauthors ${ }^{5}$ found a significant improvement in median overall survival (in their series 24.0 months for adjuvant patients vs 18.0 months in those without further therapy, $P=.03$ ), along with independent reductions in the overall mortality hazard.

Current guidelines from the National Comprehensive Cancer Network recommend that patients with an R0 resection and positive lymph nodes undergo surveillance (if squamous cell histology) or consideration of additional chemotherapy (if adenocarcinoma), whereas patients with R1 resection be considered for further chemoradiation therapy regardless of histologic type. ${ }^{6}$ In our analysis of persistent node-positive patients with $\mathrm{R} 0$ resection, patients with esophageal squamous cell cancer and patients with adenocarcinoma receiving adjuvant chemotherapy experienced improved median overall survival, suggesting that such patients should be considered for additional therapy.

In our analysis, receiving induction chemoradiation therapy, rather than induction chemotherapy, was associated with the largest decrease in the likelihood of receiving adjuvant chemotherapy. On further investigation of this relationship, it was seen that patients receiving induction chemoradiation therapy had a lower R1 or greater resection rate and fewer positive lymph nodes, with no difference in length of stay, 30-day readmission, or 30-day mortality. This may suggest that patients receiving induction chemoradiation with persistently positive nodal burden are less likely to receive adjuvant chemotherapy based on a clinical perception of decreased residual disease burden, rather than the inability to receive it because of prolonged length of stay (presumably due to postoperative complications) or early mortality. ${ }^{6}$

The differences in response rates between induction regimens have been well documented in clinical trials. Although no completed randomized controlled trial has compared induction chemotherapy with chemoradiation therapy directly, the results from studies comparing each modality with surgery alone have demonstrated substantial differences in pathologic complete response (pCR) rates. Although trials of induction chemotherapy and surgery reported pCR rates of $3 \%$ to $13 \%$, those studies evaluating induction chemoradiation and surgery achieved pCR rates of $29 \%$ (from the CROSS trial) and $40 \%$ (CALGB 9781). ${ }^{2,-10}$ Although our analysis examined patients with persistent positive nodes, it is not surprising that even among this population there was overall less disease burden seen among those patients who received induction 
chemoradiation therapy. Of note, a single high-volume institutional review in which the majority of patients received induction chemotherapy $(>90 \%)$ followed by esophagectomy found that more than $60 \%$ of their patients had 1 or more positive lymph nodes on final pathology. ${ }^{11}$ Of note, Stiles and coauthors ${ }^{11}$ did not detect a significant difference in 2-year overall survival by adjuvant therapy status.

Another line of inquiry in this analysis was whether or not the ratio of positive to examined nodes would influence whether or not a patient would receive adjuvant therapy. It would seem plausible that a patient with 15 of 15 positive lymph nodes (PEN ratio $=1$ ) could have a larger benefit from adjuvant chemotherapy than a patient with 1 of 15 positive lymph nodes (PEN ratio $=0.1$ ) because of the assumption that the positive nodal disease has likely been cleared in the latter patient. Previous literature examining increasing PEN ratio values has documented associations with poorer survival outcomes, not only in esophageal cancer but also in gastric and colon cancers, suggesting it is more tightly correlated with survival outcomes than traditional nodal categories because it adjusts for the number of nodes sampled. ${ }^{12-14}$ Our analysis demonstrated that significantly more patients receiving adjuvant therapy had N2 or N3 disease and significantly higher PEN ratios. Of interest, however, is the finding that of the 86 patients in this analysis with a PEN ratio of 1 , only $13(15.1 \%)$ received adjuvant therapy. Conversely, we did continue to detect an improvement in overall survival for patients with a relatively low burden of nodal disease (patients with a single positive lymph node and patients with a PEN ratio $<0.10$ ), suggesting a possible role for adjuvant therapy regardless of nodal burden.

\section{Study Limitations}

There are certainly limitations to this analysis. Although we compared patients on the basis of adjuvant therapy status, we are not able to document the agents administered or the total number of cycles given in the NCDB. Protocols for adjuvant chemotherapy for patients with persistent disease are available through the National Comprehensive Cancer Network, but we are not able to discern what proportion of the patients receiving adjuvant therapy were receiving care in accordance with these guidelines. ${ }^{6}$ Furthermore, although we examined Charlson-Deyo comorbidity score, inpatient length of stay, and readmission status as surrogates for postoperative "fitness" in comparing adjuvant chemotherapy status, we acknowledge that this is not inclusive. More granular data on postoperative complication rates (esophageal leak, reoperation status) or functional status after surgery (discharge location type, nutritional status) would have helped us understand likely differences between these 2 populations in a more detailed fashion. Other variables that would be helpful, but difficult to assess in any large-scale database, are those that could address the relative declines in performance measures and quality of life after esophagectomy. Previous work has demonstrated that declines in health-related quality of life (HRQL) measures, including physical, emotional, cognitive, and social effects, may persist from 6 months to 3 years after esophagectomy, possibly affecting the ability to accept additional therapy. ${ }^{15}$ Our analysis did demonstrate that increasing length of stay was independently associated with a decreased likelihood of receiving adjuvant therapy. Previous work by Nafteux and coauthors ${ }^{16}$ has shown that patients with shorter than median length of stay after esophagectomy had significantly better HRQL scores at 3 and 12 months than those patients with a higher than median hospital course. ${ }^{16}$ This could suggest a relationship between length of stay and both HRQL and ability to take on adjuvant chemotherapy. An additional limitation was that more than $60 \%$ of patients in this NCDB analysis were missing the method of surgical approach (open, laparoscopic, robotic, or converted), and therefore we did not think that this variable was reliable for analysis.

Finally, we recognize that by comparing median overall survival, rather than cancer-specific survival, the analysis is likely limited by uncaptured comorbidities influencing both the uptake of additional chemotherapy and the ultimate cause of death. Previous research in the Society of Thoracic Surgeons General Thoracic Surgery Database has found that variables independently associated with major morbidity after esophagectomy included a history of congestive heart failure, coronary artery disease, peripheral vascular disease, increasing American Society of Anesthesiology classification, diabetes, smoking status, hypertension, and steroid use. ${ }^{17}$

\section{CONCLUSIONS}

This analysis of the NCDB found that patient treatment and tumor characteristics influence the likelihood of receiving adjuvant chemotherapy for node-positive disease after induction therapy and esophagectomy. Receipt of adjuvant chemotherapy in this setting was associated with an increase in median overall survival at each pathologic nodal stage and was independent of other factors. Additional collaborative work is needed on a multi-institutional level to further identify and analyze factors that allow nodepositive patients to safely receive adjuvant chemotherapy after induction therapy and esophagectomy and evaluate its impact on both cancer-specific and overall survival. 


\section{Webcast}

You can watch a Webcast of this AATS meeting presentation by going to: https://aats.blob.core.windows.net/ media/16AM/2016-5-18/Wednesday/05-18-16_Ballroom_ IV_0741_Broderick-800.mp4.

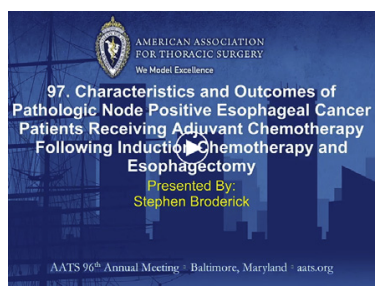

\section{Conflict of Interest Statement}

Authors have nothing to disclose with regard to commercial support.

\section{References}

1. National Cancer Institute. Surveillance, Epidemiology, and End Results Program. SEER Fact Sheets: Esophageal Cancer. Available at: http://seer.cancer. gov/statfacts/html/esoph.html. Accessed March 25, 2016.

2. Korst RJ, Kansler AL, Port JL, Lee PC, Kerem Y, Altorki NK. Downstaging of T or $\mathrm{N}$ predicts long-term survival after preoperative chemotherapy and radical resection for esophageal carcinoma. Ann Thorac Surg. 2006;82:480-5.

3. Speicher PJ, Wang X, Englum BR, Ganapathi AM, Yerokun B, Hartwig MG, et al. Induction chemoradiation therapy prior to esophagectomy is associated with superior long-term survival for esophageal cancer. Dis Esophagus. 2015; 28:788-96.

4. Shapiro J, van Lanschot JJ, Hulshof MC, van Hagen P, van Berge Henegouwen MI, Wijnhoven BPL, et al. Neoadjuvant chemoradiotherapy plus surgery versus surgery alone for oesophageal or junctional cancer (CROSS): long-term results of a randomized controlled trial. Lancet Oncol. 2015;16: 1090-8.

5. Brescia AA, Broderick SR, Crabtree TD, Puri V, Musick JF, Bell JM, et al. Adjuvant therapy for positive nodes after induction therapy and resection of esophageal cancer. Ann Thorac Surg. 2016;101:200-10.

6. National Comprehensive Cancer Network. NCCN Guidelines: Esophageal and Esophagogastric Junction Cancers. Version 3.2015. Available at: http://www. nccn.org. Accessed March 27, 2016.

7. Kelson DP, Ginsberg R, Pajak TF, Sheahan DG, Gunderson L, Mortimer J, et al. Chemotherapy followed by surgery compared with surgery alone for localized esophageal cancer. N Engl J Med. 1998;339:1979-84.

8. Darton SJ, Archer VR, Stocken DD, Mulholland PJ, Casson AG, Ferry DR, et al Preoperative mitomycin, ifosfamide, and cisplatin followed by esophagectomy in squamous cell carcinoma of the esophagus: pathologic complete response induced by chemotherapy leads to long-term survival. J Clin Oncol. 2003;21: 4009-15.

9. van Hagen P, Hulshof MC, van Lanschott JJ, Steyerberg EW, van Berge Henegouwen MI, Wijnhoven BP, et al. Preoperative chemoradiotherapy for esophageal or junctional cancer. N Engl J Med. 2012;366:2074-84.

10. Tepper J, Krasna MJ, Niedzwiecki D, Hollis D, Reed CE, Goldberg R, et al. Phase III trial of trimodality therapy with cisplatin, fluorouracil, radiotherapy, and surgery compared with surgery alone for esophageal cancer: CALGB 9781. J Clin Oncol. 2008;26:1086-92.

11. Stiles BM, Christos P, Port JL, Lee PC, Paul S, Saunders J, et al. Predictors of survival in patients with persistent nodal metastases after preoperative chemotherapy for esophageal cancer. J Thorac Cardiovasc Surg. 2010;139:387-94.

12. Samson P, Puri V, Broderick S, Patterson GA, Meyers B, Crabtree T. Extent of lymphadenectomy is associated with improved overall survival after esophagectomy with or without induction therapy. Ann Thorac Surg. 2017;103:406-15.

13. Zhang BY, Yuan J, Cui ZS, Li ZW, Li XH, Lu YY. Evaluation of the prognostic value of metastatic lymph node ratio for gastric cancer. Am J Surg. 2014;207: 555-65.
14. Tong LL, Gao P, Wang ZN, Song YX, Xu YY, Sun Z, et al. Can lymph node ratio take the place of $\mathrm{pN}$ categories in the UICC/AJCC TNM classification system for colorectal cancer? Ann Surg Oncol. 2011;18:2453-60.

15. Djarv T, Lagergren J, Blazeby JM, Lagergren P. Long-term health-related quality of life following surgery for oesophageal cancer. Br J Surg. 2008;95:1121-6.

16. Nafteux P, Durnez J, Moons J, Coosemans W, Decker G, Lerut T, et al. Assessing the relationships between health-related quality of life and postoperative length of hospital stay after oesophagectomy for cancer of the oesophagus and the gastro-oesophageal junction. Eur J Cardiothorac Surg. 2013;44:525-33.

17. Wright CD, Kucharczuk JC, O'Brien SM, Grab JD, Allen MS, STS General Thoracic Surgery Database. Predictors of major morbidity and mortality after esophagectomy for esophageal cancer: a Society of Thoracic Surgeons General Thoracic Surgery Database risk adjustment model. J Thorac Cardiovasc Surg. 2009;137:587-95.

Key Words: esophageal cancer, chemotherapy, esophagectomy, lymph nodes

\section{Discussion}

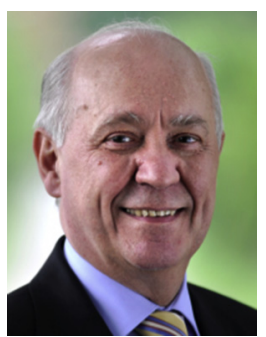

Dr Antoon E.M.R. Lerut (Leuven, Belgium). This is an important and timely topic for which little data are available, and therefore it is a controversial topic. Of course, it is well known that patients with positive nodes after induction therapy is a group with a very negative prognosis, but your study, and this is the main message from your study, suggests a survival benefit for patients who received adjuvant chemotherapy. But, as you already showed in the slides, in the adjuvant group there is a trend of patients being younger, a mean of 3 years, having less comorbidities and a shorter postoperative length of stay, and this suggests a selection bias favoring the adjuvant therapy group.

I have 4 questions for you. Intuitively, one would expect that patients who don't show any response at all will be resistant to any further chemotherapy regimen in contrast to patients who do show a partial response, and thus, again, this has the potential for inducing a selection bias. Were you able to look into this aspect of groups who had no response versus a partial response?

Dr Stephen Broderick (Baltimore, $M d$ ). I agree with you that makes sense. The short answer is no, we do not have any data regarding the response. I think you are right, that those who don't respond in the neoadjuvant setting are probably less likely to respond to adjuvant chemotherapy. Unfortunately, we cannot discern that from the NCDB. We also can't tell what agents were given, so we don't know if the adjuvant chemotherapy was simply more cycles of what was given in the neoadjuvant setting or did they attempt second-line chemotherapy or targeted therapy or anything else.

Dr Lerut. To be able to receive at least 2 cycles of chemotherapy, patients, of course, must be fit enough to survive at least for a few months after surgery, let's say about 6 months, until the end of the 2 cycles. Looking at the 
overall survival curve, visually it strikes me that a substantial fraction of that survival benefit that you mentioned, I would guess about $10 \%$, comes from within the first 6 months, again, inducing the potential for bias. Therefore, would it not have been better to take as the starting point for survival 3 months instead of 30 days? We all know that 90day mortality is a more accurate cutoff point than the 30-day mortality to evaluate the true postoperative mortality.

Dr Broderick. We were able to look into that at the 90day time point. There were 185 deaths between 30 and 90 days. I think that going forward in studies like this, the 90-day time point to begin the survival analysis, sort of accounting for that immortal period of time in the adjuvant group from 0 to 30 or 0 to 90 days, makes a lot of sense. So we did that analysis and found some difference. The difference remained statistically significant for all patients who were node-positive and highly significant in the those with N1. In the N2 and N3 groups, that $P$ value changed from .001 to .1 or .2 in the N3 group. So we lose some of that in those with N2 and N3. We also looked at the 6-month time point, beginning the survival analysis at 6 months, but we started to lose patients who were in the adjuvant group who had an early mortality on adjuvant therapy. So there were patients who started chemotherapy but died within 6 months of surgery, and so we didn't continue that out through the whole survival analysis.

Dr Lerut. That is part of my next question, because in the same context, of course, how many patients started chemotherapy but dropped out after 1 cycle, which is then the question relating to the intention-to-treat analysis. You partially already responded, but were you able to figure out how many patients dropped out after 1 cycle?

Dr Broderick. We cannot figure it out from this. Hopefully, if we have several centers with a number of patients, we will get fewer patients but more detail. You are coded as having received adjuvant therapy in the database only if you complete 2 cycles. A patient who had 1 cycle and dropped out is in the no-adjuvant group.

Dr Lerut. That is the downside of a big national database system. Let me come to the N2 and the N3 disease because their survival is only up to 3 years and not up to 5 years because there were no survivors. That would mean that, in my mind, these are just palliative patients and questioning the indication for adjuvant chemotherapy at all, whereas those with $\mathrm{N} 1$ probably have been responders and, as you showed and as you said in your subanalysis, are benefiting the most from adjuvant therapy. I think that is another message to further investigate.

Dr Broderick. That is probably true, yes.

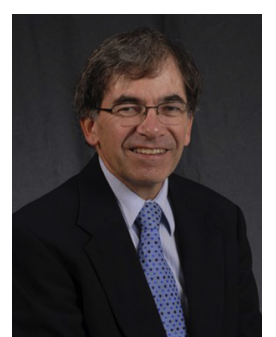

Dr Thomas M. Egan (Chapel Hill, $N C$ ). Great presentation. Perhaps you could get some insight into those other factors by analyzing the patients who did not get chemotherapy based on their income level, insurance status, and all the other covariables. Perhaps that had a significant impact on survival irrespective of the postoperative chemotherapy.

Dr Broderick. It certainly may. That is an interesting point. 